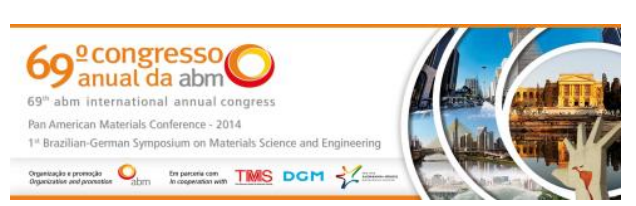

Tema: Materiais cerâmicos, compósitos e poliméricos

\title{
ANÁLISE DA VIDA ESTRUTURAL DE TUBOS DE PLÁSTICO REFORÇADO COM FIBRAS DE VIDRO (PRFV) DESTINADOS ÀS APLICAÇÕES DE REDES DE ADUÇÃO DE ÁGUA COM EXPECTATIVA MÍNIMA DE CINQUENTA ANOS*
}

\begin{abstract}
Anderson Nogueira Vidal ${ }^{1}$ Ivete Peixoto Pinheiro ${ }^{2}$

\section{Resumo}

Os tubos de Plástico Reforçado com Fibras de Vidro, também conhecidos como tubos de PRFV são pouco utilizados em obras de infraestrutura no Brasil, principalmente por desconhecimento técnico da sua vida estrutural. Este trabalho tem como objetivo verificar a expectativa mínima de 50 (cinquenta) anos de utilização dos tubos de PRFV. A metodologia adotada consiste na realização de ensaios mecânicos de longa duração em corpos de prova de tubos de PRFV por mais de 10.000 horas (1,4 anos), conforme rigor da norma ASTM D 2992 Procedimento B. Este ensaio é comumente conhecido como ensaio de HDB (Hydrostatic Design Basis). Os resultados mostraram que a metodologia utilizada é adequada para verificar e certificar a vida estrutural dos tubos de PRFV, bem como evidenciou que o valor de HDB encontrado é satisfatório e compatível, quando comparado aos outros ensaios de HDB realizados no mundo em tubos de PRFV fabricados pelo mesmo processo produtivo de enrolamento contínuo.
\end{abstract}

Palavras-chave: Plástico reforçado com fibras de vidro; Compósitos; ASTM D-2992; HDB resistência à pressão hidrostática interna de longa duração.

\section{ANALYSIS OF THE STRUCTURAL LIFE OF FIBERGLASS REINFORCED PIPE (FRP) FOR THE USES OF POTABLE WATER SUPPLY NETWORKS WITH LIFE EXPECTED MINIMUM FIFTY YEARS}

\section{Abstract}

Fiberglass Reinforced Plastic Pipe, also known as FRP pipes are rarely used in infrastructure in Brazil, mainly because absence of technical knowledge of its structural life. This paper aims to verify the minimum expectation of 50 (fifty) years of use of FRP pipes. The methodology adopted consists in the realization of long-term mechanical tests on specimens of FRP pipes for more than 10,000 hours (1.4 years), with the rigor of the standard ASTM D 2992 - Procedure B. This test is commonly known as HDB test (Hydrostatic Design Basis). The results showed that the methodology is appropriate to verify and certify the structural life of FRP pipes, and showed that the value of HDB found satisfactory and consistent when compared to other HDB test performed worldwide in FRP pipes manufactured by the same production process of continuous winding.

Keywords: Fiberglass reinforced plastic; Composite; ASTM D-2992; HDB Hydrostatic design basis.

1 Engenheiro Industrial Mecânico, Mestrando em Engenharia de Materiais, Centro Federal de Educação Tecnológica de Minas Gerais, Belo Horizonte, MG, Brasil.

2 Engenheira Química, Profa. Doutora, DEMAT, Centro Federal de Educação Tecnológica de Minas Gerais, Belo Horizonte, MG, Brasil.

\footnotetext{
* Contribuição técnica ao $69^{\circ}$ Congresso Anual da ABM - Internacional e ao 14ํㅡㄹ ENEMET - Encontro Nacional de Estudantes de Engenharia Metalúrgica, de Materiais e de Minas,21 a 25 de julho de 2014, São Paulo, SP, Brasil.
} 


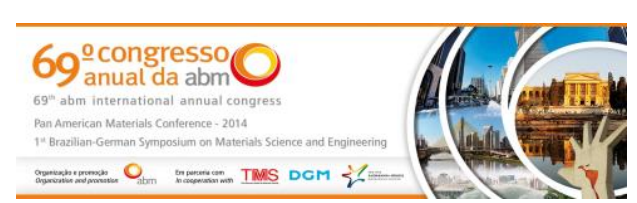

\section{INTRODUÇÃO}

Materiais diversos estão sendo empregados na fabricação de tubulações de redes de adução de água tais como o PVC (Policloreto de vinila), PEAD (Polietileno de alta densidade), aço, ferro fundido dúctil e PRFV (Plástico Reforçado com Fibras de Vidro). Por isso, em função da alta concorrência de diversos tipos de materiais de tubulações no mercado, torna-se necessário estudar a vida estrutural de tubos de Plástico Reforçado com Fibras de Vidro (PRFV) destinados às redes de adução de água com expectativa mínima de 50 (cinquenta) anos de utilização. Os tubos de PRFV geralmente são produzidos pelo processo de enrolamento contínuo (Filament Winding) para a referida aplicação, onde as fibras de vidro são impregnadas com resina termofixa de poliéster e enroladas sobre o mandril rotatório [1]. O compósito é a classe de material heterogêneo e multifásico, em que um dos componentes, descontínuo, é responsável pela principal resistência ao esforço (componente estrutural) e o outro, contínuo, é o meio de transferência deste esforço (componente matricial). A interface entre estes materiais tem característica dominante sobre suas características individuais [2]. Executar os ensaios mecânicos de longa duração para os tubos de PRFV (10.000 horas) conforme a norma ASTM D 2992 Procedimento B (Hydrostatic Design Basis) [3] de modo a contemplar o mínimo de 18 (dezoito) pontos de falhas, gerar o gráfico $\log x \log$ de "alongamento circunferencial x tempo", definir a reta de regressão e extrapolar para 50 (cinquenta) anos é o método científico para verificar e certificar que este produto atende ao requisito de expectativa mínima de 50 (cinquenta) anos de vida estrutural, conforme previsto na norma AWWA C-950 [4].

A relevância do trabalho em questão promoverá amplo entendimento técnico da vida estrutural de tubos de PRFV com expectativa mínima de 50 (cinquenta) anos, visando propiciar, principalmente, a sua ampla utilização em obras de infraestrutura no Brasil, tais como as redes de adução de água, visto que os tubos de PRFV são muito competitivos em preços entre os diâmetros que variam de 400 a $3600 \mathrm{~mm}$, comprimentos de 6 a 18 metros, pressões de até 2,5 MPa e classes de rigidez de até 20.000 Pascal. Desse modo espera-se também estimular os pesquisadores no processo de aperfeiçoamento e de avanço da tecnologia PRFV no Brasil em consonância com o atendimento dos exigentes requisitos técnicos de qualidade deste produto.

\section{MATERIAIS E MÉTODOS}

As fibras de vidro utilizadas são do tipo ECR (Owens Corning) e foram utilizadas na forma como foram adquiridas. Na fabricação dos tubos de PRFV foram utilizadas a resina termofixa poliéster PD 3535 (Reichhold), pré-acelerada com Octoato de Cobalto (6\%), catalisada com o Mekp DM 50. Foram fabricados os corpos de provas, ou seja, tubos de PRFV com diâmetro de $300 \mathrm{~mm}$, espessura de $3,5 \mathrm{~mm}$ e comprimento de $1000 \mathrm{~mm}$. A fabricação foi realizada por enrolamento contínuo, utilizando equipamento de tecnologia Flowtite, 0 qual combina enrolamento circunferencial de fibras de vidro contínuas superior a $70^{\circ}$ conjugado com a adição de fibras de vidro picadas ao longo da espessura do tubo, conforme figuras 1 e 2 .

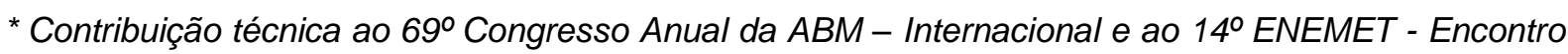
Nacional de Estudantes de Engenharia Metalúrgica, de Materiais e de Minas,21 a 25 de julho de 2014, São Paulo, SP, Brasil.
} 

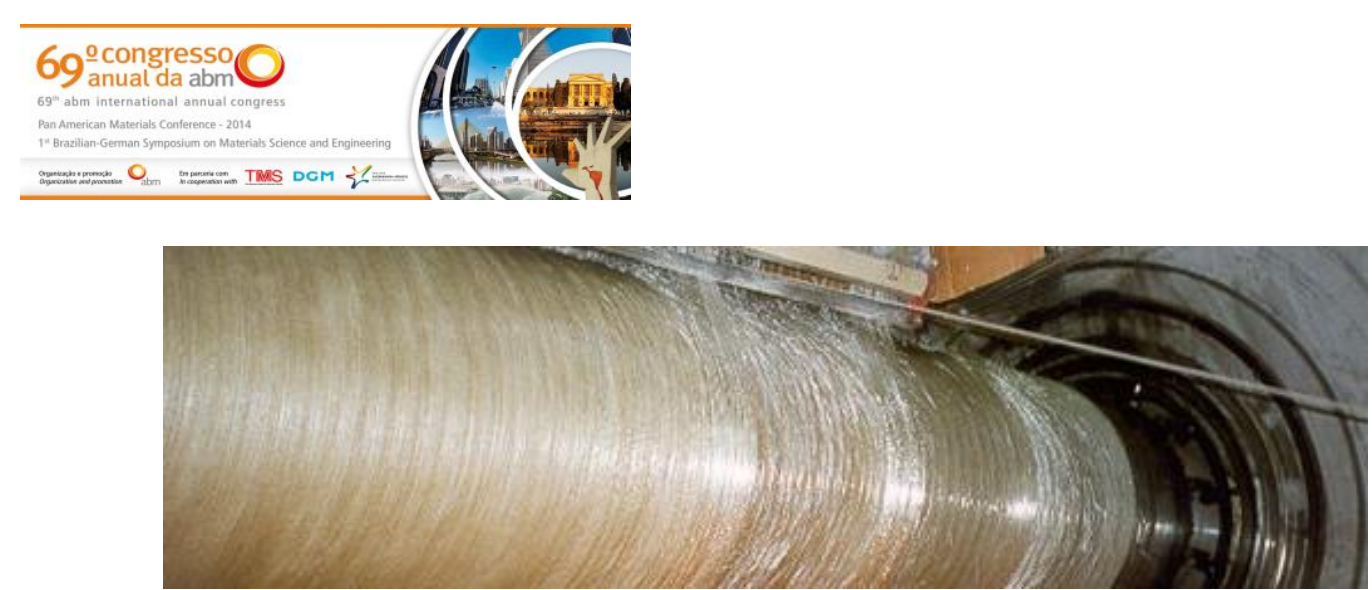

Figura 1. Processo de enrolamento contínuo (Tecnologia Flowtite) de fabricação de tubos PRFV

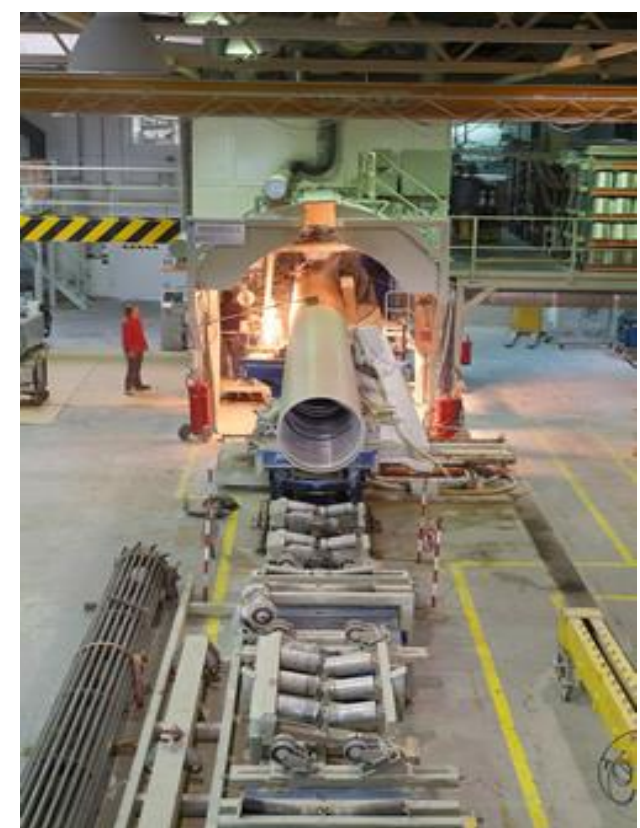

Figura 2. Visão geral do processo de enrolamento contínuo (Tecnologia Flowtite) de fabricação de tubos PRFV

Os ensaios de HDB (Hydrostatic Design Basis) ou também conhecido como "resistência à pressão hidrostática interna de longa duração" foram realizados em 24 (vinte e quatro) corpos de provas de acordo com a norma ASTM D-2992 Procedimento B em um laboratório no Brasil (Empresa "A") especificamente montado para os ensaios de HDB, conforme figura 3 , devidamente climatizado com 2 (dois) equipamentos de ar condicionado (12 horas de funcionamento contínuo cada) e com todos os 12 (doze) equipamentos individualizados e devidamente calibrados (12 (doze) manômetros e 12 (doze) cronômetros). É importante salientar que os ensaios foram acompanhados por organismo independente DNV (Det Norske Veritas), o qual verificou e testemunhou a conformidade integral do ensaio com a norma supracitada.

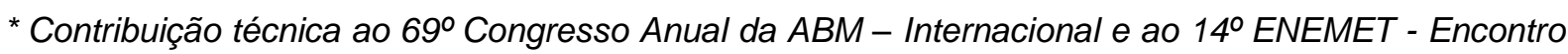
Nacional de Estudantes de Engenharia Metalúrgica, de Materiais e de Minas,21 a 25 de julho de 2014, São Paulo, SP, Brasil.
} 

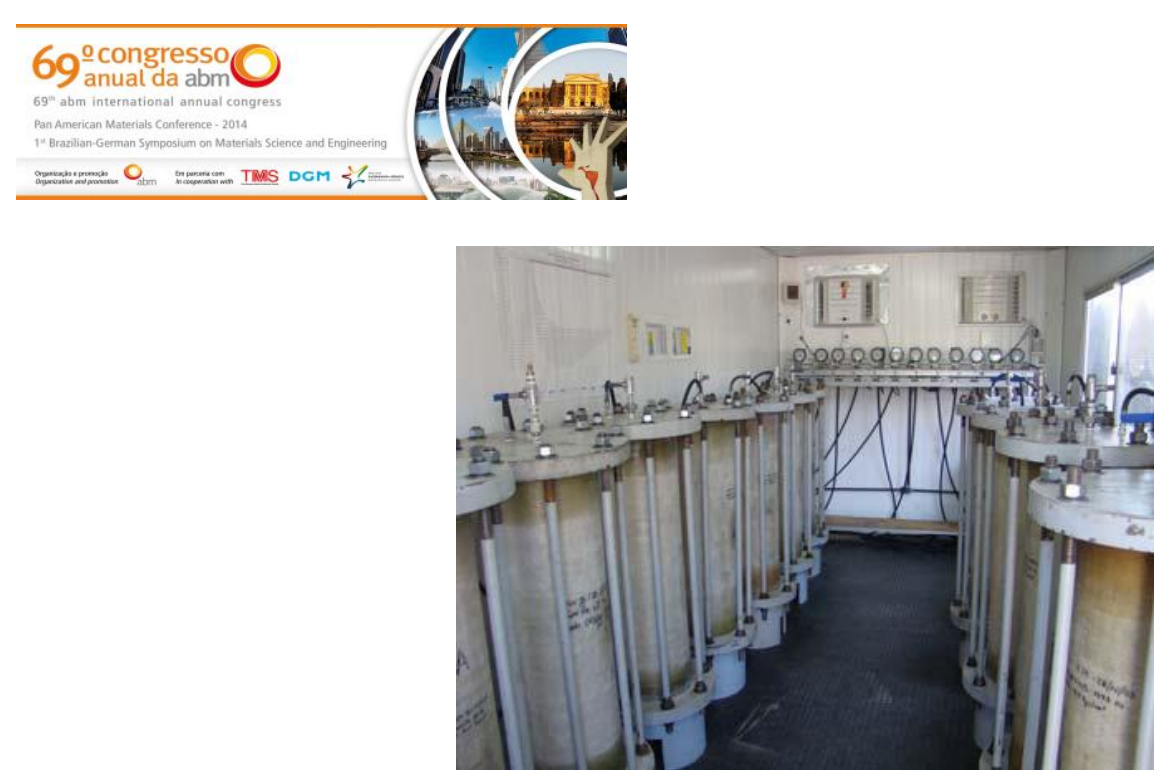

Figura 3. Laboratório de ensaio de HDB (Hydrostatic Design Basis) no Brasil para tubos PRFV

Inicialmente foram ensaiados 12 (doze) corpos de prova simultaneamente. Os ensaios consistiram na avaliação dos efeitos em tubos de PRFV cheios de água, os quais foram individualmente submetidos à pressão hidrostática determinada e especificada conforme tabela 1, a qual permaneceu constante ao longo de todo 0 ensaio até a ocorrência da falha por vazamento, também conhecida como exsudação. Os corpos de provas mais solicitados falham primeiro. Cada pressão hidrostática especificada e utilizada durante o ensaio, por sua vez, gerou um diferente valor de tração circunferencial com 0 respectivo alongamento circunferencial em cada tubo, o qual consequentemente falhou em diferente tempo por vazamento (exsudação) durante a realização do ensaio. Esses tempos de falhas foram anotados e registrados. À medida que os corpos de prova falhavam, os mesmos foram substituídos por novos corpos de provas montados nos dispositivos de ensaios de HDB e iniciados novos ensaios conforme previsto na tabela 1.0 ensaio completo teve a duração máxima de 13.396 horas e gerou 24 (vinte e quatro) pontos de falhas estatisticamente válidos. Os pontos de falhas foram plotados em gráfico $\log x \log$ de alongamento circunferencial $x$ tempo, os quais foram ajustados para uma reta de regressão, de modo que o alongamento circunferencial de falha foi extrapolado para 438.000 horas, ou seja, foi extrapolado para 50 (cinquenta) anos (longo prazo), conforme descrito nas normas ASTM D-2992 [3] e AWWA-C-950 [4], o qual foi utilizado para o cálculo do HDB.

Os equipamentos utilizados no laboratório foram:

a) Água.

b) Sistema de pressurização capaz de aplicar uma pressão hidrostática interna constante e uniforme no corpo de prova. O sistema é composto por bomba hidropneumática Magral 10 MPa e manifold, conforme figura 3. O sistema é capaz de atingir a pressão de ensaio sem excedê-la e mantê-la na tolerância especificada durante a duração do ensaio.

c) Manômetro Classe A1 para leitura de pressão;

d) Cronômetro COEL - E520 integrado ao sistema de pressurização, capaz de medir o tempo de falha com exatidão de $\pm 2 \%$;

e) Dispositivo de fechamento das extremidades dos corpos de provas, conforme figura 3, capazes de permitir ou impedir a movimentação longitudinal durante o ensaio e que suportem as pressões atingidas. Os dispositivos não devem transmitir esforços aos corpos de provas.

\footnotetext{
* Contribuição técnica ao $69^{\circ}$ Congresso Anual da ABM - Internacional e ao 14ํㅡㄹ ENEMET - Encontro Nacional de Estudantes de Engenharia Metalúrgica, de Materiais e de Minas,21 a 25 de julho de 2014, São Paulo, SP, Brasil.
} 


\section{RESULTADOS E DISCUSSÃO}

As falhas dos corpos de provas de HDB foram caracterizadas como exsudação, ou seja, vazamentos ocorridos pelas passagens de água através das microfissuras formadas na interfase vidro-resina quando os tubos estavam pressurizados. Os resultados dos ensaios de HDB (Hydrostatic Design Basis) estão demonstrados na tabela 1.

Tabela 1. Resultado do ensaio de HDB (Hydrostatic Design Basis) para tubos PRFV

\begin{tabular}{|c|c|c|c|}
\hline Falha & $\begin{array}{c}\text { Alongamento } \\
(\%)\end{array}$ & $\begin{array}{l}\text { Pressão } \\
\left(\mathrm{kgf} / \mathrm{cm}^{2}\right)\end{array}$ & $\begin{array}{l}\text { Tempo real da } \\
\text { falha (Horas) }\end{array}$ \\
\hline 01 & 1,115 & 53,00 & 2,96 \\
\hline 02 & 1,115 & 53,00 & $2.739,11$ \\
\hline 03 & 1,115 & 53,00 & $2.498,85$ \\
\hline 04 & 1,115 & 53,00 & $3.427,49$ \\
\hline 05 & 1,103 & 52,50 & $1.166,68$ \\
\hline 06 & 1,103 & 52,50 & 478,26 \\
\hline 07 & 1,094 & 52,00 & 555,06 \\
\hline 08 & 1,094 & 52,00 & $2.943,29$ \\
\hline 09 & 1,094 & 52,00 & $2.567,50$ \\
\hline 10 & 1,083 & 51,50 & 49,50 \\
\hline 11 & 1,083 & 51,50 & 71,18 \\
\hline 12 & 1,073 & 51,00 & 963,88 \\
\hline 13 & 1,073 & 51,00 & $4.788,40$ \\
\hline 14 & 1,062 & 50,50 & 22,84 \\
\hline 15 & 1,062 & 50,50 & $4.958,63$ \\
\hline 16 & 1,030 & 49,00 & $5.997,48$ \\
\hline 17 & 0,988 & 47,00 & $7.871,61$ \\
\hline 18 & 0,966 & 46,00 & $8.802,20$ \\
\hline 19 & 0,945 & 45,00 & $11.193,03$ \\
\hline 20 & 0,924 & 44,00 & $13.127,46$ \\
\hline 21 & 0,914 & 43,50 & $13.222,39$ \\
\hline 22 & 0,903 & 43,00 & $6.998,47$ \\
\hline 23 & 0,882 & 42,00 & $10.195,11$ \\
\hline 24 & 0,872 & 41,50 & $13.396,35$ \\
\hline
\end{tabular}

A tabela 2 mostra que os números de experimentos de HDB realizados comparados aos números de experimentos mínimos (18 (dezoito) corpos de provas) por faixa de tempos de falhas em horas foi integralmente cumprido e em total conformidade com a norma ASTM D-2992 - Procedimento B.

\footnotetext{
* Contribuição técnica ao 69ำ Congresso Anual da ABM - Internacional e ao 14ํㅡㄹ ENEMET - Encontro Nacional de Estudantes de Engenharia Metalúrgica, de Materiais e de Minas,21 a 25 de julho de 2014, São Paulo, SP, Brasil.
} 


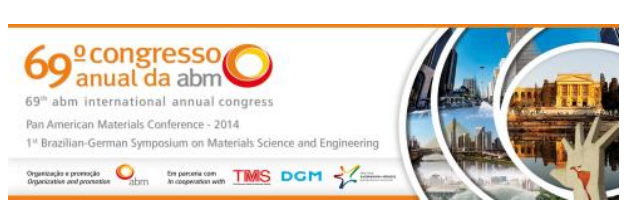

Tabela 2. Números de experimentos de HDB realizados comparados aos números de experimentos mínimos por tempo de falha em horas

\begin{tabular}{|c|c|c|c|}
\hline $\begin{array}{c}\text { Tempo de falha } \\
\text { (horas) }\end{array}$ & $\begin{array}{c}\text { Número mínimos de } \\
\text { falhas exigidas no ensaio } \\
\text { de HDB }\end{array}$ & $\begin{array}{c}\text { Número de falhas } \\
\text { obtidas no ensaio de } \\
\text { HDB }\end{array}$ & Resultado \\
\hline 10 a $1.000 \mathrm{~h}$ & 04 & 07 & Satisfatório \\
\hline 1.000 a $6.000 \mathrm{~h}$ & 03 & 09 & Satisfatório \\
\hline Após $6.000 \mathrm{~h}$ & 03 & 08 & Satisfatório \\
\hline Após $10.000 \mathrm{~h}$ & 01 & 05 & Satisfatório \\
\hline $\begin{array}{c}\text { Ensaio } \\
\text { completo }\end{array}$ & 18 & 24 & Satisfatório \\
\hline
\end{tabular}

A figura 4 e a tabela 3 mostram que a análise dos dados de HDB foi realizada pelo método dos mínimos quadrados para o alongamento circunferencial, utilizando-se o tempo como variável dependente e estabeleceu para 438.000 horas (50 anos) com $95 \%$ de limite inferior de confiança o valor de HDB de $0,784 \%$ para alongamento. Os requisitos da norma ASTM D-2992- Procedimento B foram cumpridos integralmente, assegurando a validade dos dados. O coeficiente de correlação ( $r$ ) obtido foi 0,561 . Este valor de correlação quando comparado ao mínimo permitido de 0,514 foi satisfatório, conforme previsto na norma ASTM D-2992.

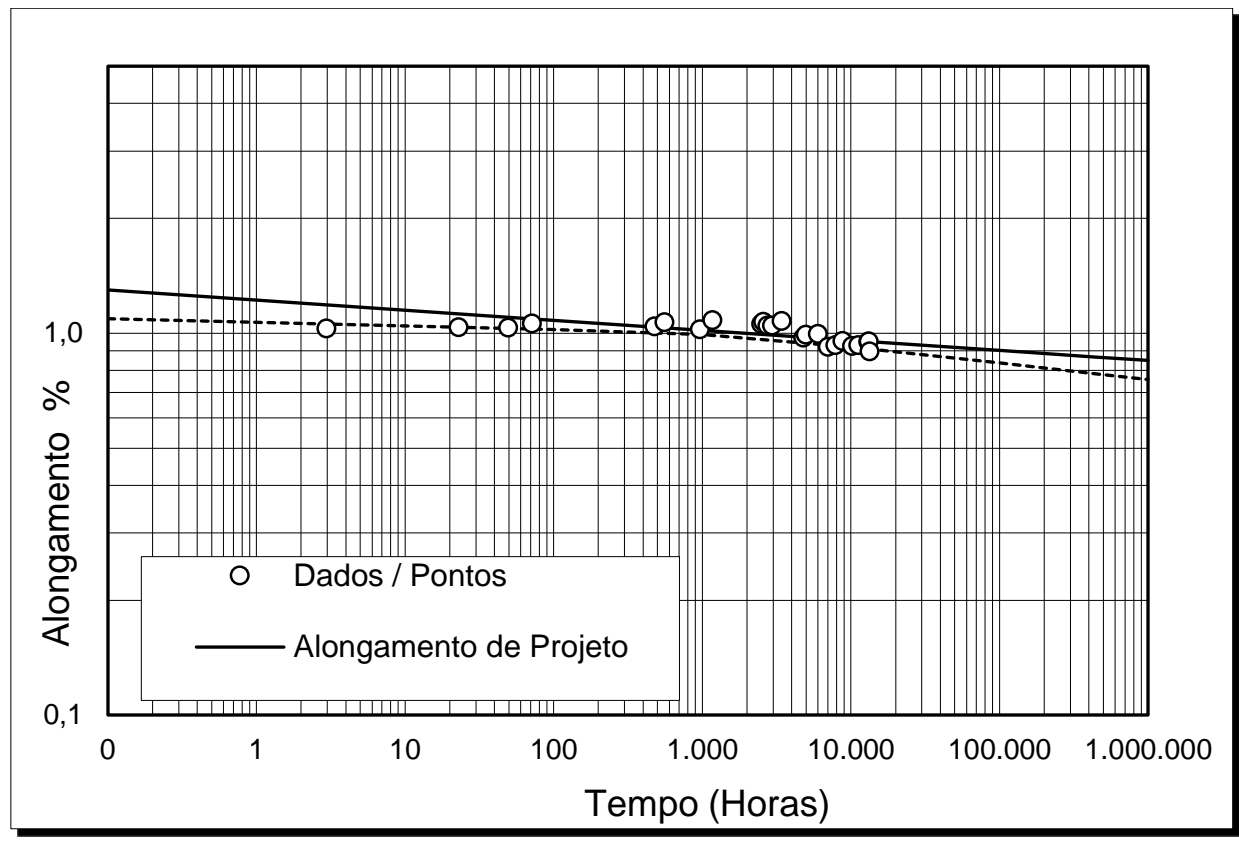

Figura 4. Gráfico relativo ao cálculo do HDB (Hydrostatic Design Basis) pelo método dos mínimos quadrados

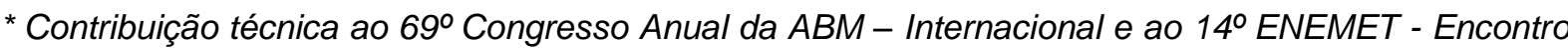
Nacional de Estudantes de Engenharia Metalúrgica, de Materiais e de Minas,21 a 25 de julho de 2014, São Paulo, SP, Brasil.
} 


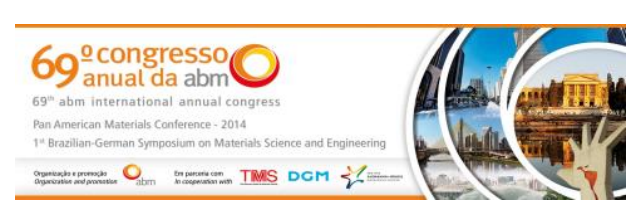

Tabela 3. Cálculo do HDB (Hydrostatic Design Basis) pelo método dos mínimos quadrados com 95\% de limite inferior de confiança

\begin{tabular}{|c|c|c|}
\hline $\begin{array}{c}\text { Alongamento Projetado } \\
(\%)\end{array}$ & $\begin{array}{l}\text { Tempo } \\
\text { ( Horas ) }\end{array}$ & $\begin{array}{l}95 \% \text { Limite inferior de Confiança } \\
\text { (Alongamento Projetado (\%)) }\end{array}$ \\
\hline 1,297 & 0,1 & 1,093 \\
\hline 1,081 & 100 & 1,024 \\
\hline 1,018 & 1.000 & 0,992 \\
\hline 0,958 & 10.000 & 0,921 \\
\hline 0,901 & 100.000 & 0,836 \\
\hline 0,888 & 175.200 & 0,816 \\
\hline 0,867 & 438.000 & 0,784 \\
\hline 0,848 & 1.000 .000 & 0,757 \\
\hline \multicolumn{3}{|c|}{ HDB = Alongamento de Projeto (50 anos) / Coeficiente de segurança AWWA C-950 } \\
\hline $\begin{array}{c}\text { Alongamento projetado de } \\
\text { HDB (\%) (Admissível) }\end{array}$ & Tempo (Horas) & 95\% Limite inferior de Confiança \\
\hline $\begin{array}{r}\mathrm{HDB}=0,867 / 1,8 \\
\mathrm{HDB}=0,482\end{array}$ & 438.000 & $\begin{array}{r}\text { HDB }(95 \% \text { LCL })=0,784 / 1,8 \\
\text { HDB }(95 \% \text { LCL })=0,436\end{array}$ \\
\hline
\end{tabular}

Foi desenvolvida e obtida a equação de regressão de HDB em função de alongamento, conforme a equação (1), pelo método dos mínimos quadrados.

$$
\log (\text { Alongam } .)=0,0866-0,0263 \times \log (\text { Tempo })
$$

Analogamente foi desenvolvida e obtida a equação de regressão de HDB em função de tempo, conforme a equação (2), pelo método dos mínimos quadrados.

$$
\log (\text { Tempo })=3,287-37,951 \times \log (\text { Alongam } .)
$$

O resultado alcançado no ensaio de HDB de 0,784\% de alongamento para 438.000 horas (50 anos) com 95\% de limite inferior de confiança é compatível e está ligeiramente superior aos resultados encontrados na literatura, quando comparado aos valores obtidos por outras pesquisas no mundo. Pearson [5] obteve o resultado no ensaio de HDB de $0,63 \%$ de alongamento para 438.000 horas (50 anos) com $95 \%$ de limite inferior de confiança através de ensaios realizados na empresa Owens Corning, em 1988, na Bélgica, conforme a norma ASTM D 2992 - Procedimento B, utilizando-se o total de 27 corpos de provas com comprimento de 2 metros e diâmetro de $600 \mathrm{~mm}$. Posteriormente Pearson [6] obteve novo resultado no ensaio de HDB de 0,65\% através de ensaios realizados na empresa Flowtite, em 1997, na Noruega, utilizando-se o total de 44 corpos de provas com comprimento de 1 metro para diâmetro de $150 \mathrm{~mm}$ e comprimento de 2 metros para diâmetro de $600 \mathrm{~mm}$. As referências e as publicações de resultados são escassas, pois se tratam de ensaios caros em função da montagem do laboratório, aquisição de equipamentos e instrumentação, números de corpos de provas e acompanhamento permanente por funcionários por mais de 10.000 horas ininterruptas, os quais são normalmente realizados pelos fabricantes de tubos de PRFV e não publicados na literatura.

\footnotetext{
* Contribuição técnica ao $69^{\circ}$ Congresso Anual da ABM - Internacional e ao 14ํㅡㄹ ENEMET - Encontro Nacional de Estudantes de Engenharia Metalúrgica, de Materiais e de Minas,21 a 25 de julho de 2014, São Paulo, SP, Brasil.
} 


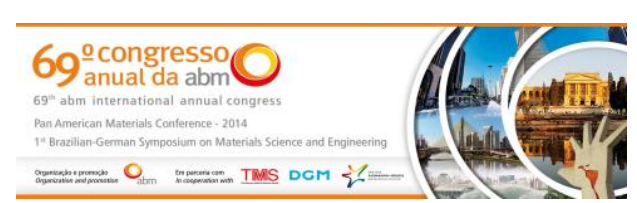

\section{CONCLUSÃo}

Todos os requisitos da norma ASTM D-2992 - procedimento B foram cumpridos e a validação dos dados foi satisfatória, bem como a metodologia utilizada para desenvolver os ensaios de HDB (Hydrostatic Design Basis) com o rigor da referida norma ASTM D-2992 - procedimento B mostrou-se adequada, pois resultou em valor de HDB com característica satisfatória e compatível, quando comparado aos outros ensaios de HDB realizados no mundo em tubos de PRFV fabricados pelo mesmo processo produtivo.

Foi possível evidenciar que os corpos de provas, laboratório e instrumentações para os ensaios de HDB garantiram as condições ideais provendo confiabilidade para a execução dos mesmos, além da conformidade com os requisitos normativos. Salienta-se que o acompanhamento das etapas iniciais, intermediárias e finais ocorreram de formas satisfatórias, conforme verificação do organismo Certificador Internacional (DNV - Det Norske Veritas).

A viabilidade técnica dos tubos de PRFV e o entendimento técnico da vida estrutural dos mesmos com a expectativa mínima de 50 (cinquenta) anos, possivelmente promoverá maior utilização dos tubos de PRFV em obras de infraestrutura no Brasil, tais como as redes de adução de água em substituição aos demais materiais empregados na fabricação de tubulações de saneamento básico (PVC, PEAD, aço e ferro fundido dúctil), principalmente quando os diâmetros das tubulações forem iguais ou superiores a $300 \mathrm{~mm}$.

\section{Agradecimentos}

Ao Engenheiro Antônio Carvalho que colaborou no auxílio técnico e na execução da pesquisa.

\section{REFERÊNCIAS}

1 Auter $\mathrm{G}$ et al. Compósitos I: materiais, processos, aplicações, desempenhos e tendências. São Paulo, São Paulo: ABMACO, 2008.

2 Associação Brasileira De Normas Técnicas. ABNT NBR 15536-1, 2007: sistemas para adução de água, coletores-tronco, emissários de esgoto sanitário e águas pluviais: tubos e conexões de plástico reforçado de fibra de vidro (PRFV) - parte 1: tubos e juntas para adução de água, 2007.

3 American Society for Testing And Materials. ASTM D 2992 - 2006: Standard practice for obtaining hydrostatic or pressure design basis for fiberglass (Glass-Fiber-Reinforced Thermosetting-Resin) pipe and fittings (metric). Annual Book of ASTM Standards, 2006.

4 American Water Works Association. AWWA C-950 - 2007: standard fiberglass pressure pipe, 2007.

5 Pearson LE. Hydrostatic Design Basis (HDB) - owens-corning pressure pipe - report number T-88-103-V. Owens-Corning Test Report - Owens-Corning, Bruxelles. 1988.

6 Pearson LE. Hydrostatic Design Basis (HDB) - flowtite pressure pipe - report number T-95-101-R. Flowtite Test Report - Flowtite Technology AS, Sandefjord. 1997. 4p

\footnotetext{
* Contribuição técnica ao $69^{\circ}$ Congresso Anual da ABM - Internacional e ao 14ํㅡㄹ ENEMET - Encontro Nacional de Estudantes de Engenharia Metalúrgica, de Materiais e de Minas,21 a 25 de julho de 2014, São Paulo, SP, Brasil.
} 